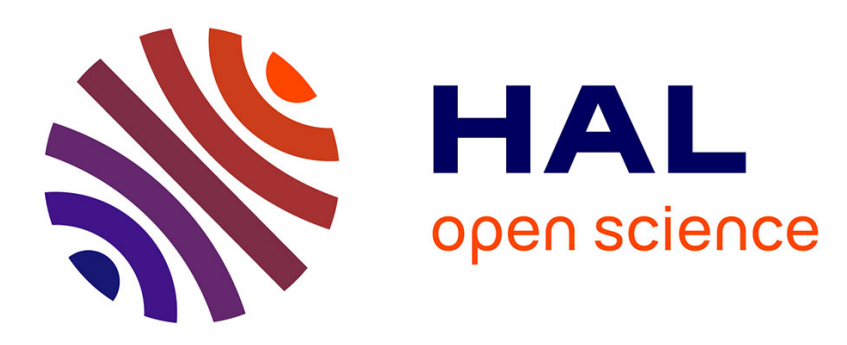

\title{
Recommandations françaises de la prise en charge et du traitement de l'ostéoporose masculine
}

\author{
Béatrice Bouvard, Karine Briot, Erick Legrand, Hubert Blain, Véronique \\ Breuil, Roland Chapurlat, Marc Duquenne, Pascal Guggenbuhl, Eric \\ Lespessailles, Thierry Thomas, et al.
}

\section{To cite this version:}

Béatrice Bouvard, Karine Briot, Erick Legrand, Hubert Blain, Véronique Breuil, et al.. Recommandations françaises de la prise en charge et du traitement de l'ostéoporose masculine. Revue du Rhumatisme, 2021, 88 (3), pp.173-182. 10.1016/j.rhum.2021.02.024 . hal-03237497

\section{HAL Id: hal-03237497 \\ https://hal-univ-paris.archives-ouvertes.fr/hal-03237497}

Submitted on 10 Jun 2021

HAL is a multi-disciplinary open access archive for the deposit and dissemination of scientific research documents, whether they are published or not. The documents may come from teaching and research institutions in France or abroad, or from public or private research centers.
L'archive ouverte pluridisciplinaire HAL, est destinée au dépôt et à la diffusion de documents scientifiques de niveau recherche, publiés ou non, émanant des établissements d'enseignement et de recherche français ou étrangers, des laboratoires publics ou privés. 
Recommandations françaises de la prise en charge et du traitement de l'ostéoporose masculine

Béatrice Bouvard ${ }^{\mathrm{a} *}$, Karine Briot ${ }^{\mathrm{b}}$, Erick Legrand ${ }^{\mathrm{a}}$, Hubert Blain ${ }^{\mathrm{c}}$, Véronique Breuil ${ }^{\mathrm{d}}$, Roland Chapurlat $^{\mathrm{e}}$, Marc Duquenne ${ }^{\mathrm{f}}$, Pascal Guggenbuhl ${ }^{\mathrm{g}}$, Eric Lespessailles ${ }^{\mathrm{h}}$, Thierry Thomas ${ }^{\mathrm{i}}$, Bernard Cortet ${ }^{j}$

${ }^{a}$ Service de rhumatologie, CHU d'Angers, 4 rue Larrey, 49933 Angers Cedex 9 et Unité GEROM UPRES EA 4658 - Université d'Angers

${ }^{\mathrm{b}}$ Service de rhumatologie, Hôpital Cochin, Université Paris Descartes, Paris, 75014

${ }^{\mathrm{c}}$ Pôle de gérontologie, CHU et Université de Montpellier, 34090

${ }^{\text {d } U n i v e r s i t e ́ ~ C o ̂ t e ~ d ' A z u r, ~ S e r v i c e ~ d e ~ r h u m a t o l o g i e, ~ C H U ~ d e ~ N i c e ~-~ H o ̂ p i t a l ~ P a s t e u r ~} 2$ - 30 Voie Romaine - CS 51069 - 06001 Nice Cedex 1 - France

e INSERM UMRS_1033, Université de Lyon, Service de rhumatologie, Hôpital E Herriot, Lyon, 69437

${ }^{\mathrm{f}} \mathrm{CH}$ Saumur, 100, Route de Fontevraud, 49400 Saumur

g Université de Rennes, INSERM, INRA, CHU Rennes, Institut NUMECAN (Nutrition Metabolisms and Cancer), UMR 1241, F-35000 Rennes, France

${ }^{\mathrm{h}}$ Service de rhumatologie, CHR Orléans, 14 avenue de l'hôpital, 45067 Orléans, France

${ }^{i}$ Service de rhumatologie, Hôpital Nord, CHU Saint-Etienne, Saint-Etienne, France et INSERM U1059, Université de Lyon-Université Jean Monnet, Saint-Etienne, France

${ }^{j}$ Service de rhumatologie et ULR 4490, CHU, Lille et Université Lille, 59000

*Auteur correspondant : Béatrice Bouvard, Service de rhumatologie, CHU d'Angers, 4 rue Larrey, 49933 Angers Cedex 9

bebouvard@chu-angers.fr

Tel : 0241353576

Fax : 0241356014

Résumé

Objectifs : nous proposons des recommandations destinées à tous les médecins amenés à prendre en charge des hommes ayant une ostéoporose ou à risque d'une telle maladie.

Méthodes : le contenu de ces recommandations a été rédigé en conformité avec la méthode HAS et validé par un groupe de travail. Lorsque les données de la littérature étaient insuffisantes, les recommandations ont été basées sur un accord professionnel prenant en compte l'état des pratiques et les opinions d'experts. Les recommandations ont été soumises à un groupe de lecture.

Résultats : nous proposons 15 recommandations détaillant la population cible, les moyens d'évaluation, le traitement et le suivi. Nous insistons sur les points suivants 1) une fracture spontanée ou provoquée par un traumatisme mineur doit faire rechercher une maladie osseuse fragilisante 2) le risque de fracture et de mortalité après fracture sévère augmente avec l'âge, rapidement à partir de 70-75 ans 3) les indications thérapeutiques dépendent de la présence ou non d'une fracture, du type de fracture (sévère/non sévère) et de la valeur la plus basse du Tscore de la DMO 4) l'évaluation du risque de chute et sa prise en charge doivent faire partie de la prise en charge de l'ostéoporose 5) le suivi clinique est indispensable pour adapter la prise en charge à l'évolution du risque de fracture. 
Conclusions : ces premières recommandations françaises, que nous avons souhaitées proches de celles concernant les femmes ménopausées, visent à améliorer le dépistage et la prise en charge de l'ostéoporose masculine.

Mots-clés : recommandations, ostéoporose, homme, fracture, densité minérale osseuse

\section{Points essentiels}

- Premières recommandations françaises sur la prise en charge et le traitement de l'ostéoporose masculine

- Le risque de fracture et de mortalité après fracture sévère augmente avec l'âge, rapidement à partir de 70-75 ans

- Les indications thérapeutiques dépendent de la présence ou non d'une fracture, du type de fracture et de la valeur la plus basse du T-score

- L'évaluation du risque de chute et sa prise en charge doivent faire partie de la prise en charge de l'ostéoporose

\section{Introduction}

Entre 20 à $25 \%$ des fractures ostéoporotiques cliniques surviennent chez l'homme. Après 50 ans, un homme a une probabilité d'environ $20 \%$ d'avoir une fracture ostéoporotique avant la fin de sa vie [1]. Le concept de fracture sévère s'applique à l'homme chez qui un excès de mortalité, quel que soit l'âge, est 2 à 3 fois supérieur à la mortalité observée dans la même tranche d'âge en l'absence de fracture, pour les fractures ostéoporotiques vertébrales, de hanche mais également du bassin, du fémur distal et de l'humérus proximal [2-4]. L'excès de mortalité après une fracture sévère est important dans les 3 premiers mois et perdure jusqu'à 10 ans après une fracture de hanche. En France, en 2008-2009, toutes causes confondues, $33 \%$ des hommes de 55 ans ou plus sont morts dans l'année qui a suivi une fracture de l'extrémité supérieure du fémur (FESF), dont $20 \%$ au cours des trois premiers mois (https ://drees.solidaritessante.gouv.fr/etudes-et-statistiques/publications/etudes-et-resultats/article/quel-risque-dedeces-un-an-apres-une-fracture-du-col-du-femur). Parmi les survivants, 10 à $25 \%$ seront définitivement institutionnalisés après fracture vertébrale, FESF, ou fracture du bassin $[5,6]$. L'impact des fractures ostéoporotiques sur la qualité de vie est durable [7].

L'antécédent de fracture est un facteur de risque bien connu de récidive fracturaire (risque relatif multiplié par 2) et son caractère récent est important à considérer dans l'évaluation du risque fracturaire $[8,9]$.

Malgré la fréquence de l'ostéoporose chez l'homme et ses conséquences, les données récentes en France du Système National des Données de Santé (SNDS) de la Caisse Nationale de l'Assurance Maladie des Travailleurs Salariés (CNAMTS) ont confirmé la faible prise en charge de l'ostéoporose après une fracture. Ainsi, parmi les 
155810 patients de plus de 50 ans hospitalisés pour une fracture en France en 2012, hommes et femmes confondus, seuls $5 \%$ ont eu une ostéodensitométrie dans l'année suivant la fracture, et $15 \%$ un traitement anti-ostéoporotique spécifique. Pourtant $50 \%$ d'entre eux ont reçu une supplémentation en calcium et/ou en vitamine D soulignant le fait que la pathologie osseuse avait été identifiée mais sa prise en charge largement inadaptée. Ainsi l'événement fracture, qui rend pourtant visible la maladie, modifie peu la prise en charge de l'ostéoporose de ces patients. Les fractures chez les hommes sont à tort considérées le plus souvent comme traumatiques sans nécessité de réaliser un bilan de fragilité osseuse ni de proposer un traitement antiostéoporotique spécifique [10] et leurs conséquences sont sous-estimées et mal connues.

\section{Méthodes}

Ces premières recommandations françaises portant sur l'ostéoporose masculine sont destinées à tous les médecins amenés à prendre en charge des hommes ayant une ostéoporose ou à risque d'une telle maladie. Les objectifs sont d'exposer les éléments clés de l'évaluation des patients à risque élevé de fracture et les principes de prise en charge de cette ostéoporose. Ces recommandations tiennent compte des indications actuelles et des conditions de remboursement des traitements, des examens biologiques et de l'imagerie en France. Le contenu de ces recommandations a été rédigé en conformité avec la méthode de la Haute Autorité de Santé (https://www.has-sante.fr/upload/docs/application/pdf/2020-

02/reco363_gm_rbp_maj_janv_2020_cd_2020_01_22_v0.pdf) et validé par un groupe de travail. Lorsque les données de la littérature étaient insuffisantes, les recommandations ont été basées sur un accord professionnel prenant en compte l'état des pratiques et les opinions d'experts. Ces recommandations ne peuvent pas envisager tous les cas particuliers et ne peuvent se substituer à la responsabilité individuelle du médecin vis-à-vis de son patient. Ces recommandations sont élaborées à l'initiative du Groupe de Recherche et d'Information sur les Ostéoporoses (GRIO) et de la Société Française de Rhumatologie (SFR). Elles ont été écrites par un chargé de projet et un comité scientifique, discutées et relues par un comité de lecture pluridisciplinaire. Outre le GRIO et la SFR, plusieurs sociétés savantes : Société Française de Chirurgie Orthopédique, Société Française d'Endocrinologie, Société Française de Gériatrie et de Gérontologie et Société Française d'Urologie ont participé à l'élaboration et à la révision du texte et ont validé ces recommandations.

\section{Recommandations}

\subsection{Qui évaluer ? (Recommandations 1 et 2)}


Recommandation 1. Après la survenue d'une fracture spontanée ou provoquée par un traumatisme mineur, il est recommandé de rechercher une maladie osseuse fragilisante (Grade A).

Une fracture de fragilité osseuse survient en l'absence de traumatisme ou suite à un traumatisme de faible énergie comme une chute de sa hauteur.

Les fractures par fragilité osseuse peuvent survenir sur tous les os, à l'exception du crâne, de la face, des vertèbres au-dessus de T4, de l'avant pied et des orteils, de la main et des doigts.

Les fractures vertébrales situées au-dessus de T4 doivent faire rechercher d'autres maladies osseuses (tumeurs, infections).

Recommandation 2. Il est recommandé de rechercher les facteurs de risque d'ostéoporose chez l'homme après $\mathbf{7 0}$ ans et de réaliser une mesure de densité minérale osseuse quel que soit l'âge dès lors qu'il existe un ou plusieurs de ces facteurs (Accord professionnel).

L'âge est un facteur de risque indépendant d'ostéoporose et de fracture [11]. L'incidence des fractures chez l'homme augmente rapidement à partir de 70-75 ans. L'âge de survenue de la fracture est aussi un important facteur de risque de décès après fracture, en particulier pour les FESF.

En tenant compte de ces données et des recommandations de la plupart des organisations internationales (IOF, NOF [12], ISCD), nous recommandons de rechercher les facteurs de risque d'ostéoporose chez l'homme après 70 ans et de mesurer la densité minérale osseuse (DMO) chez l'homme dès lors qu'il existe un ou plusieurs facteurs de risque de fragilité osseuse (encadrés 1 et 2) (Accord professionnel). L'ostéoporose masculine est souvent multifactorielle et le risque de fracture ostéoporotique augmente avec le cumul des facteurs de risque d'ostéoporose [13]. Les pathologies les plus fréquemment associées à une ostéoporose chez les hommes sont les maladies inflammatoires avec corticothérapie prolongée, l'hypogonadisme spontané ou iatrogène, la maladie alcoolique, la bronchopneumopathie chronique obstructive. La corticothérapie [14] et l'hormonothérapie anti-androgénique [15] font l'objet de recommandations françaises spécifiques récemment publiées et ne seront pas détaillées ici.

\subsection{Comment évaluer ? (Recommandations de 3 à 7$)$}

Recommandation 3. Le diagnostic d'ostéoporose repose sur la recherche des antécédents de fracture(s) non vertébrale(s) et la réalisation d'une imagerie du rachis dès lors qu'il existe des signes cliniques ou anamnestiques évocateurs de fractures vertébrales (Accord professionnel). 
Le principal facteur de risque de fracture est l'antécédent personnel de fracture par fragilité osseuse [16-18]. Le caractère récent de la fracture est important à prendre en compte, $20 \%$ des patients ont une deuxième fracture dans l'année suivant la première fracture et $1 / 3$ dans les 2 ans (concept du risque de fracture à court terme ou « imminent risk of fracture ») [9]. Le risque relatif de nouvelle fracture (après une première fracture) est le même dans les 2 sexes, augmente avec l'âge et le fait d'avoir un T-score $\leq-2,5[8,9]$. Le risque de nouvelle fracture reste élevé jusqu'à 10 années après la fracture initiale durant lesquelles $60 \%$ des hommes restant en vie vont de nouveau avoir une fracture. Les antécédents parentaux (surtout maternels) de fractures majeures et de FESF sont également associés à une augmentation du risque de fracture chez les hommes [19, 20].

Il est recommandé de réaliser une imagerie vertébrale dans l'évaluation d'une ostéoporose à la recherche d'une fracture prévalente dans les situations suivantes (position officielle de l'International Society for Clinical Densitometry 2019) :

- un épisode de rachialgies aiguës même transitoire et résolutif,

- un antécédent rapporté mais non documenté de fracture vertébrale,

- une perte de taille historique $>4 \mathrm{~cm}$ (mesure de la taille comparée à la taille rapportée à l'âge de 20 ans),

- la prise d'une corticothérapie $\geq 5 \mathrm{mg}$ d'équivalent prednisone par jour depuis au moins 3 mois.

Le nombre et le grade (degré de réduction de hauteur de la vertèbre) des fractures vertébrales sont à considérer pour évaluer la sévérité de l'ostéoporose [21].

L'imagerie vertébrale à réaliser en première intention est la radiographie standard du rachis lombaire et thoracique de face et de profil. Le diagnostic de fracture vertébrale est retenu en cas de diminution de l'une des hauteurs vertébrales de plus de $20 \%$ selon la méthode semiquantitative de Genant [22].

Le dépistage des fractures vertébrales peut être également réalisé par la technique appelée «Vertebral Fracture Assessment » (VFA), qui est une imagerie vertébrale acquise lors de la réalisation de la densitométrie osseuse. Cette imagerie, permettant de voir de face et de profil les vertèbres de T4 à L5, est beaucoup moins irradiante que les radiographies standard. Les fractures vertébrales prévalentes objectivées sur la VFA sont associées à une augmentation du risque de fracture incidente de vertèbre, de hanche, et de fracture ostéoporotique majeure [23]. La découverte d'une fracture vertébrale sur la VFA devra s'accompagner de la réalisation d'une radiographie standard afin de ne pas méconnaître le caractère éventuellement tumoral de la fracture que ne permet pas de déterminer la seule VFA.

L'analyse de l'imagerie vertébrale est rendue plus difficile chez l'homme comparée à la femme par l'existence de déformations vertébrales plus fréquentes, notamment au rachis dorsal, en lien avec la pathologie arthrosique et particulièrement aux séquelles d'ostéodystrophie rachidienne de croissance qui peuvent notamment donner des déformations cunéiformes régulières et souvent multi-étagées des corps vertébraux.

Le FRAX ${ }^{\circledR}$ (fracture risk assessment, www.sheffield.ac.uk/FRAX) est un outil permettant de prédire, en valeur absolue, le risque de FESF et le risque de fracture « majeure » (hanche, vertèbre clinique, avant-bras distal, épaule) à 10 ans [24]. L'outil FRAX ${ }^{\circledR}$ a été développé en utilisant principalement des cohortes féminines, les hommes représentant $25 \%$ de l'ensemble 
des sujets inclus, c'est-à-dire 15000 hommes. Son usage est à réserver aux situations pour lesquelles la décision de traiter ou de ne pas traiter est difficile à prendre. Dans ce cas, le seuil d'intervention choisi est fonction de l'âge. Le même seuil d'intervention est appliqué aux hommes et aux femmes, puisque l'efficacité et le rapport coût-efficacité d'une intervention chez les hommes sont similaires à ceux des femmes pour un risque équivalent [25].

Recommandation 4. Le diagnostic d'ostéoporose et l'évaluation du risque de fracture ostéoporotique incluent la mesure de DMO mesurée par absorptiométrie biphotonique à rayons-X au rachis lombaire et à l'extrémité supérieure du fémur, en utilisant une courbe de référence masculine pour calculer le T-score (Accord professionnel).

L'ostéodensitométrie par absorptiométrie biphotonique à rayons-X (DXA) est la technique de référence pour mesurer la DMO au rachis lombaire et à l'extrémité supérieure du fémur. La DXA est remboursée par l'Assurance Maladie, sous conditions, depuis le $1^{\text {er }}$ juillet 2006 (encadré 2).

Chez l'homme de 50 ans ou plus, cible majoritaire du dépistage, le résultat est exprimé en valeur absolue (densité surfacique, $\mathrm{g} / \mathrm{cm}^{2}$ ) et à l'aide du T-score qui exprime l'écart entre la densité osseuse du patient et la densité osseuse moyenne observée chez les hommes jeunes, en bonne santé, entre 20 et 30 ans (encadré 3).

Le choix de la courbe de référence pour calculer le T-score reste débattu [26]. En France, nous recommandons d'utiliser une courbe de référence masculine plutôt que féminine pour les raisons suivantes :

- utiliser une courbe de référence masculine permet de dépister un plus grand nombre de patients à risque de fracture ; ainsi en utilisant la courbe masculine NHANES III $6 \%$ des hommes ont un T-score $\leq-2,5$ contre $4 \%$ en utilisant la courbe féminine NHANES III [27], ce qui cadre mieux avec les données épidémiologiques fracturaires,

- les études portant sur les traitements anti-ostéoporotiques chez l'homme ont toutes utilisé des courbes de référence masculines.

Il est recommandé de mesurer la DMO à deux sites, le rachis lombaire et l'extrémité supérieure du fémur. La mesure à l'extrémité supérieure du fémur (la hanche totale et/ou le col fémoral) est plus fiable car elle est moins soumise aux artefacts que la mesure au rachis lombaire et elle est mieux corrélée au risque de fracture. Pour chaque diminution d'un écart type de DMO à la hanche le risque de fracture de hanche est multiplié par environ 3 [17, 28, 29].

Le Trabecular Bone Score (TBS) est un indice de texture osseuse obtenu à partir d'une image de DMO de la colonne lombaire. Il est corrélé à des paramètres de microarchitecture osseuse et donne des informations complémentaires à la mesure de la DMO au rachis lombaire, en termes de prédiction des fractures. La mesure systématique du TBS n'est pas recommandée dans la prédiction du risque de fracture et le suivi des traitements car sa capacité à classer les patients n'est pas suffisamment établie. Après ajustement sur le score FRAX et les fractures vertébrales prévalentes, le TBS est modestement associé aux fractures majeures et fractures de hanche chez les hommes [30, 31]. Son usage est à réserver aux situations pour lesquelles la décision de traiter ou ne pas traiter est difficile à prendre. Le seuil de décision thérapeutique du FRAX-TBS est dans ce cas le même que celui du FRAX ${ }^{\circledR}$. 


\section{Recommandation 5. L'évaluation du risque de fracture doit inclure l'évaluation du} risque de chute (Accord professionnel).

Quatre-vingt-dix pour cent des FESF sont précédées d'une chute. On estime qu'un tiers des sujets de plus de 65 ans et la moitié de ceux de plus de 85 ans font une ou plusieurs chutes par an. L'incidence des chutes est moins élevée chez les hommes que chez les femmes (sex-ratio $\mathrm{H} / \mathrm{F}$ 0,35). Chez les sujets de plus de 65 ans, cinq à dix pour cent des chutes sont responsables de fractures, dont une fois sur trois de l'extrémité supérieure du fémur. Les sujets particulièrement à risque sont ceux ayant chuté dans la dernière année, ayant peur de tomber ou se sentant instables. Chez ceux-ci, il est recommandé de quantifier le nombre de chutes et les blessures, de repérer les troubles de l'équilibre (incapacité à tenir en appui unipodal plus de 5 secondes), de la marche (plus de 5 secondes pour faire 4 mètres) et de la force musculaire (incapacité de se relever d'une chaise 5 fois sans les mains en 15 secondes)[32].

Recommandation 6. Devant la découverte d'une fracture par fragilité osseuse ou d'une densité osseuse basse ( $T$-score $<-3$ ), il est recommandé de réaliser en première intention le bilan biologique suivant : calcémie, albuminémie, phosphatémie, créatininémie (clairance estimée), électrophorèse des protéines sériques, hémogramme, CRP, 25(OH) vitamine $\mathrm{D}$, phosphatases alcalines totales, transaminases, gamma GT et testostérone totale (Accord professionnel).

Ce bilan biologique minimal est indispensable pour rechercher une autre cause de fracture (myélome, métastase osseuse), pour orienter vers autre ostéopathie fragilisante (hyperparathyroïdie, ostéomalacie), ou une ostéoporose secondaire (cirrhose...) et pour vérifier l'absence de contre-indication à la mise en route d'un traitement (insuffisance rénale sévère ou terminale, hypocalcémie).

Plus le sujet est jeune plus le bilan biologique devra être exhaustif. En fonction du contexte clinique ou biologique, les dosages suivants pourront être ajoutés : ferritinémie (hémochromatose, bilan hépatique anormal), parathormone (devant une calcémie anormale), TSH, FSH, LH, prolactine, cortisol libre urinaire des 24 heures (signes cliniques d'endocrinopathie), glycémie (diabète), calciurie et phosphaturie des 24 heures (avec calcul du TRP et du TmP/GFR s'il est bas), tryptase sérique (mastocytose), et anticorps antitransglutaminases (maladie coeliaque).

Les marqueurs du remodelage osseux ne sont pas recommandés dans le bilan d'une ostéoporose ou d'une fracture, d'autre part ils ne prédisent pas le risque de fracture dans la population masculine $[33,34]$.

Recommandation 7. En l'absence de contexte étiologique, la survenue d'une fracture par fragilité osseuse est inhabituelle chez l'homme avant 70 ans, il est alors recommandé de le référer à un spécialiste des pathologies osseuses (Accord professionnel). 
Selon le contexte pourront être recherchés une cause rare et de diagnostic difficile (mastocytose, hémopathie) ou un déterminant génétique expliquant la fragilité osseuse (encadré 4).

\subsection{Comment traiter l'ostéoporose masculine ? (Recommandations de}

\section{8 à 12)}

L'objectif du traitement est de réduire le risque de fractures. La prise en charge est à la fois pharmacologique et non pharmacologique et repose sur une décision partagée entre le patient et le médecin. Le patient doit être informé sur sa maladie et les risques inhérents aux fractures ostéoporotiques, les traitements disponibles, l'intérêt de l'observance thérapeutique et du suivi de sa maladie ainsi que l'efficacité attendue et les effets indésirables possibles des traitements. Les études thérapeutiques chez l'homme sont moins nombreuses que chez la femme, avec de plus faibles effectifs et avec le plus souvent la variation de DMO pour objectif primaire et non l'incidence des fractures. Les preuves du bénéfice anti-fracturaire des médicaments de l'ostéoporose chez l'homme ont été établies dans des populations ostéoporotiques définies par des critères densitométriques ou par l'existence d'une fracture et par des études de «bridging ». Cette méthodologie, validée par les autorités de santé internationales dont l'agence européenne du médicament, est considérée comme suffisante pour obtenir l'indication de traitement des hommes à haut risque de fracture, à la condition que l'efficacité anti-fracturaire du médicament ait déjà été démontrée dans une population de femmes ostéoporotiques et que le risque de fracture des hommes ostéoporotiques inclus dans les études soit comparable à celui de cette population féminine (https://www.ema.europa.eu/en/documents/scientific-guideline/draft-guidelineevaluation-medicinal-products-treatment-primary-osteoporosis_en.pdf). C'est le cas des études portant sur l'ostéoporose masculine dans lesquelles les variations de DMO, les variations du taux des marqueurs du remodelage osseux et la diminution du risque de fracture sont superposables à celles observées dans les études thérapeutiques portant sur l'ostéoporose postménopausique (lorsque l'étude a été conçue dans cet objectif). Les études thérapeutiques sont résumées dans le tableau 1 [35-46].

Le choix de la molécule et de la voie d'administration tient compte de l'âge, des antécédents, du risque de fracture vertébrale et/ou périphérique et de la sévérité de la fracture, dans le respect des contre-indications et des conditions de remboursement.

Compte tenu du gradient de risque entre une valeur abaissée de DMO et l'augmentation du risque de fracture, les indications thérapeutiques dépendent de la valeur la plus basse du T-score au rachis lombaire et au fémur et de la sévérité de la situation clinique (présence ou non d'une fracture et type de fracture (sévère/non sévère)) (tableau 2).

Ainsi, la réalisation d'une ostéodensitométrie est recommandée avant toute décision thérapeutique (Grade A).

Recommandation 8. En présence d'une fracture sévère (vertèbre, bassin, extrémité supérieure du fémur, fémur distal, humérus proximal) par fragilité osseuse, un traitement anti-ostéoporotique est recommandé si l'un des T-score est $\leq-1$ (Accord professionnel). 
En cas de T-score lombaire et fémoral > -1, il faudra chercher une autre cause d'ostéopathie fragilisante que l'ostéoporose, à densité conservée ou augmentée. Un avis spécialisé doit être sollicité.

Recommandation 9. En présence d'une fracture non sévère (poignet, cheville notamment) par fragilité osseuse, un traitement anti-ostéoporotique est recommandé si l'un des T-score est $\leq \mathbf{- 2}$ (Accord professionnel).

Recommandation 10. En l'absence de fracture, un traitement anti-ostéoporotique est recommandé chez les hommes avec facteurs de risque de fragilité osseuse ou de chute et si l'un des T-score est $\leq-3$ (Accord professionnel).

Recommandation 11. Chez les patients ayant eu une fracture de l'extrémité supérieure du fémur par fragilité osseuse, il est recommandé d'utiliser l'acide zolédronique en première intention (Accord professionnel).

Chez l'homme, l'acide zolédronique est le seul traitement à avoir démontré une réduction du risque de fracture dans un essai randomisé contrôlé [47]. Son utilisation est également à privilégier dans les situations suivantes : troubles mnésiques suggérant une mauvaise observance, troubles digestifs majeurs (gastrectomie, malabsorption), polymédication (Accord professionnel).

Chez les hommes avec fracture sévère non vertébrale, fracture vertébrale unique ou fracture non sévère, les traitements suivants ont l'indication et sont remboursés en France :

- acide zolédronique $5 \mathrm{mg}$ en perfusion unique annuelle,

- risédronate $35 \mathrm{mg}$, en administration per os hebdomadaire.

Chez les hommes avec au moins deux fractures vertébrales, les traitements suivants ont l'indication, et sont remboursés en France :

- tériparatide $20 \mu \mathrm{g}$, en injection sous-cutanée quotidienne,

- acide zolédronique $5 \mathrm{mg}$ en perfusion unique annuelle,

- risédronate $35 \mathrm{mg}$, en administration per os hebdomadaire.

Dans cette situation, le tériparatide peut être prescrit en première intention au moment du diagnostic en l'absence de contre-indication (Accord professionnel). Le tériparatide est alors prescrit pour une durée de 18 mois. Il doit être suivi par une prescription de bisphosphonates per os ou iv.

En cas de contre-indication à ces traitements, telle qu'une insuffisance rénale, il est utile de demander l'avis d'un expert en pathologie osseuse.

Le dénosumab a une indication dans le traitement de l'ostéoporose chez l'homme, dans le traitement de la perte osseuse associée à un traitement anti androgénique chez les hommes atteints de cancer de la prostate et dans le traitement de l'ostéoporose induite par les glucocorticoïdes. Il n'est pas remboursé dans ces indications. 
En cas de découverte d'un hypogonadisme, hors castration pour un cancer de la prostate, le patient doit être référé à un spécialiste de la pathologie endocrinienne qui évaluera après bilan adéquat la nécessité d'un traitement androgénique.

La normalisation de la testostéronémie augmente la DMO chez les hommes ayant un hypogonadisme acquis comme un adénome à prolactine $[48,49]$. Chez les hommes âgés de plus de 65 ans présentant un hypogonadisme primaire (périphérique), le traitement androgénique augmente la DMO au rachis lombaire et à la hanche [50,51]. Aucune étude n'a évalué l'effet anti-fracturaire de la substitution androgénique. Aucune étude n'a évalué les effets combinés des bisphosphonates ou du tériparatide avec une substitution androgénique. La testostérone n'est pas un traitement de l'ostéoporose masculine et ne doit pas être prescrite au titre du traitement de l'ostéoporose chez les hommes avec une testostéronémie normale ou en monothérapie chez les hommes à haut risque de fracture [52, 53].

\section{Recommandation 12. Il est recommandé d'éliminer les facteurs de risque modifiables de fragilité osseuse, de prévenir le risque de chute, de normaliser les apports en calcium et en protéines, d'obtenir une concentration en $25(\mathrm{OH})$ vitamine D optimale et de favoriser une activité physique en charge (Accord professionnel).}

Cette recommandation définit le cadre global de la prise en charge d'un patient ostéoporotique combinant des mesures pharmacologiques détaillées plus haut et des mesures nonpharmacologiques. Il est important de convaincre le patient de l'intérêt de sa démarche proactive dans la réalisation de ces mesures avec l'objectif d'une meilleure adhésion à l'ensemble de sa prise en charge.

Cette recommandation s'applique également chez le sujet à risque mais ne relevant pas d'un traitement pharmacologique.

\subsection{1. Éviction des facteurs de risque modifiables}

L'ostéoporose masculine est multifactorielle ; il est ainsi important chaque fois que possible d'éliminer ces facteurs de risque : arrêt de l'intoxication tabagique, diminution de l'intoxication alcoolique et son interruption définitive en cas d'ostéoporose d'origine alcoolique, utilisation de la plus petite dose efficace de glucocorticoïdes et si possible leur arrêt en utilisant si nécessaire les traitements de fond permettant de réaliser une épargne cortisonique.

\subsubsection{Prévention des chutes}

Il est recommandé de poser annuellement 3 questions aux personnes âgées de 65 ans et plus (1. Êtes-vous tombé(e) dans la dernière année et si oui combien avez-vous fait de chutes et avezvous eu des blessures ? 2. Avez-vous peur de tomber ? 3. Vous sentez-vous instable en marchant ou en vous levant ?).

Les personnes répondant non aux trois questions sont à faible risque de chute. Les personnes répondant oui à une des questions doivent réaliser des tests d'équilibre, de marche et de force (cf recommandation 5). L'évaluation individuelle des personnes à risque élevé de chute doit permettre le cas échéant de corriger les facteurs de risque de chute, incluant la prise en charge 
des troubles visuels [54], le traitement d'une carence en vitamine D [55], la réduction des risques environnementaux de chute au domicile, l'adaptation du chaussage et la réduction progressive des médicaments responsables de chutes, en particulier les psychotropes, benzodiazépines [56] et les médicaments qui induisent une hypotension.

La pratique d'activités physiques comportant un entraînement spécifique visant à améliorer l'équilibre est un facteur déterminant pour diminuer le risque de chute. Il doit être associé à d'autres types d'exercices : renforcement musculaire, endurance, travail de coordination, travail des extenseurs rachidiens, augmentation des amplitudes articulaires, qui ont montré leur efficacité pour diminuer le risque de chute ou le risque de chute compliquée. L'activité physique adaptée peut être proposée en groupes en cas de risque faible ou modéré de chute ou individuellement en cas de risque élevé de chute [57, 58]. Le médecin traitant peut s'appuyer sur des réseaux de soins spécifiques (filières fractures, équipes de gériatrie, services de rééducation) en cas de risque élevé de chute [59].

Au-delà de son effet sur la diminution du risque de chute, l'exercice physique en charge a des effets bénéfiques sur la DMO à la hanche et au rachis lombaire $[60,61]$ et la pratique d'une activité physique régulière est associée à une diminution modérée du risque de fracture de hanche [62, 63]. En l'absence de recommandations spécifiques pour l'ostéoporose, nous proposons de suivre les recommandations diffusées dans le cadre du programme national nutrition santé à savoir de pratiquer l'équivalent d'au moins 30 minutes de marche rapide par jour au minimum 5 fois par semaine (Actualisation des repères du Programme national nutrition santé [PNNS] - Révisions des repères relatifs à l'activité physique et à la sédentarité 2016) (www.anses.fr).

\subsubsection{Apports en calcium}

Chez l'adulte, la référence nutritionnelle pour la population (RNP) pour les apports calciques, mise à jour en 2016, est de $950 \mathrm{mg} /$ jour chez les plus de 24 ans. Les apports alimentaires sont à privilégier (produits laitiers et eaux minérales riches en calcium) (Accord professionnel). En pratique, il est possible d'évaluer l'apport alimentaire par un auto-questionnaire fréquentiel disponible en ligne (www.grio.org). En cas d'apports alimentaires insuffisants, il doit être complété par un ajustement diététique lorsque cela est possible (par exemple en préconisant des eaux minérales riches en calcium) ou par un supplément médicamenteux en calcium avec pour objectif un apport global (alimentation plus supplémentation) de 1 gramme par jour. L'efficacité du supplément en calcium administré seul, dans le but de prévenir les fractures ostéoporotiques, n'est pas démontrée.

\subsubsection{Apports en vitamine $\mathrm{D}$}

La carence en vitamine D peut être responsable d'une hyper résorption osseuse induite par l'hyperparathyroïdie secondaire, de troubles de minéralisation et d'une augmentation du risque de chute en raison de modifications des capacités de contrôle moteur neuromusculaire [64, 65]. Il est recommandé de supplémenter en vitamine D afin d'obtenir une concentration en $25(\mathrm{OH})$ vitamine $\mathrm{D}$ comprise entre 30 à $60 \mathrm{ng} / \mathrm{mL}$ (Accord professionnel). 
La périodicité et la dose de vitamine D initiales dépendent de la concentration en $25(\mathrm{OH})$ vitamine D et de l'IMC du patient [66]. Il n'est pas recommandé d'apporter des doses $>100000$ UI de vitamine D en une prise [67] (Accord professionnel).

\subsubsection{Apports en protéines}

Un faible apport alimentaire en protéines est associé à une augmentation du risque de fracture [68]. L'augmentation de l'apport en protéines est associée à une diminution du risque de facture de hanche [69]. L'apport quotidien recommandé en protéines est de 1 gramme $/ \mathrm{kg}$ de poids corporel en particulier chez le sujet âgé.

\subsection{Quel suivi du patient ostéoporotique ? (Recommandations de 13 à} 15)

\section{Recommandation 13. Il est recommandé de réaliser au minimum un suivi clinique annuel du patient pris en charge pour une ostéoporose pour évaluer l'observance et la tolérance du traitement (Accord professionnel).}

Il comporte le recueil des événements fracturaires, la mesure de la taille, la recherche de nouveaux facteurs de risque tels que la présence de maladies et/ou traitements inducteurs d'ostéoporose, l'évaluation du risque de chutes, l'observance des traitements, la mise en pratique des mesures non pharmacologiques d'accompagnement.

Le suivi permettra également de s'assurer du bon suivi bucco-dentaire et de l'absence de signe d'intolérance médicamenteuse.

Les données de tolérance des traitements au long cours spécifiques à l'homme sont rares, la durée maximale des études randomisées étant de 3 ans. Quelques études de suivi chez l'homme sont disponibles à 7 ans pour l'alendronate [70] et à 3 ans pour le dénosumab dans une population japonaise [71]. Il est par ailleurs raisonnable de penser que la tolérance des traitements de l'ostéoporose n'est pas différente chez l'homme et chez la femme.

Le risque d'ostéonécrose de la mâchoire est faible en cas d'utilisation des antirésorbeurs pour l'ostéoporose : de l'ordre de 1 cas pour 10000 à 100000 patient-année [72], très proche de l'incidence observée dans la population générale. La Société Française de Stomatologie, Chirurgie Maxillo-Faciale et Chirurgie Orale a émis des recommandations de bonnes pratiques concernant ce risque (www.sfscmfco.fr). Ainsi, il est recommandé d'effectuer les éventuels soins dentaires nécessaires au début du traitement. Si le patient a un suivi régulier par un dentiste et en l'absence d'avulsion dentaire ou autre geste dentaire profond prévu à court terme, un traitement par antirésorbeur peut être débuté immédiatement. Si le patient n'a pas de suivi dentaire il est recommandé de consulter un dentiste. En cas de risque élevé de fracture à court terme comme après une fracture sévère, l'évaluation dentaire ne doit pas retarder la mise en route d'un traitement. Le suivi dentaire sous traitement est identique à celui recommandé pour la population générale, au minimum une fois par an. Il est possible d'effectuer des avulsions 
dentaires sous bisphosphonates lorsqu'elles sont nécessaires sous couverture antibiotique et de la façon la moins traumatisante possible. La prise de bisphosphonates ou de dénosumab pour une ostéoporose n'est pas une contre-indication à la mise en place d'un implant dentaire.

Les antirésorbeurs sont associés à une très faible augmentation du risque de fracture atypique fémorale, et ce d'autant plus que le traitement est prolongé. Le risque absolu de fracture atypique chez des patients traités par bisphosphonates est compris entre 3,2 et 50 cas pour 100 000 patients-année [73]. Le diagnostic doit être évoqué chez un patient souffrant de douleurs persistantes en charge des cuisses.

Les marqueurs du remodelage osseux, qui ne sont pas recommandés dans le bilan d'une ostéoporose ou d'une fracture, peuvent être dosés lorsqu'un traitement par bisphosphonates per os est recommandé. Ainsi, un dosage de CTX sérique (marqueur de la résorption osseuse) peut être réalisé entre les $3 \mathrm{e}$ et $12 \mathrm{e}$ mois selon le traitement [74] en respectant les conditions de réalisation de l'examen suivantes : dosage à jeun, le matin avant 9 heures, à distance d'une fracture (> 6 mois). Si le dosage de CTX sérique est élevé, il faut revoir avec le patient les conditions de prise du traitement et le cas échéant envisager un changement de traitement. La concentration de CTX peut être augmentée par l'insuffisance rénale ou l'existence d'une fracture récente.

L'évaluation morphologique du rachis à la recherche d'une fracture vertébrale (radiographies ou VFA) est indiquée chez un homme traité s'il existe des rachialgies et/ou une perte de taille prospective $>2 \mathrm{~cm}$ et/ou s'il reçoit des corticoïdes [75, 76] (Accord professionnel).

Recommandation 14. Une mesure de la DMO est recommandée dans les 2 à 3 ans suivant le début du traitement et à chaque fois qu'est envisagée une modification du traitement (arrêt ou changement) (Accord professionnel).

Le but est de vérifier au minimum l'absence de perte osseuse (définie par une diminution de plus de $0,03 \mathrm{~g} / \mathrm{cm}^{2}$ de DMO) (Grade B) [77].

Recommandation 15. Il est recommandé au-delà de 3 ans de traitement d'adapter la durée du traitement en fonction de l'âge, de l'évolution de la densité osseuse sous traitement, de la tolérance du traitement, de la gravité initiale de l'ostéoporose et de la survenue éventuelle d'une nouvelle fracture sous traitement (Accord professionnel).

La suspension d'un traitement au-delà de 3 ans peut être discutée chez un patient qui remplit toutes les conditions suivantes (Accord professionnel) :

- pas de nouvelle fracture non traumatique sous traitement,

- pas d'apparition de nouveaux facteurs de risque d'ostéoporose,

- pas de diminution significative de la DMO $>0,03 \mathrm{~g} / \mathrm{cm}^{2}$ au rachis ou à la hanche,

- en cas de fracture sévère, un T-score au col du fémur > -2,5 voire -2.

Si le patient ne remplit pas l'ensemble de ces conditions, il peut être utile de prendre l'avis d'un spécialiste de pathologie osseuse pour (a) réévaluer le diagnostic, (b) analyser le choix 
thérapeutique initial et l'observance, (c) décider de la poursuite ou du changement de traitement (Accord professionnel).

En cas de poursuite ou de modification du traitement, ou de suspension du traitement une nouvelle évaluation sera proposée 2 à 3 ans plus tard (Accord professionnel).

Une réévaluation du risque de fracture comprenant une nouvelle densitométrie osseuse est recommandée 2 ans après l'interruption du traitement (Accord professionnel). Ensuite, le délai dépendra du type de traitement. L'effet densitométrique est plus prolongé à l'arrêt de l'acide zolédronique qu'à l'arrêt des autres traitements, il n'y a pas d'effet rémanent avec le dénosumab et le tériparatide.

Compte tenu de la perte osseuse rapide et du risque augmenté de fractures vertébrales multiples à l'arrêt du dénosumab, la prescription d'un traitement par bisphosphonates est recommandée, de préférence injectable par une perfusion unique d'acide zolédronique $5 \mathrm{mg}$ ou par voie orale pour une période de 12 mois (Accord professionnel).

Compte tenu de l'absence de rémanence osseuse du tériparatide, il est recommandé de prescrire à son arrêt un bisphosphonate per os ou iv pour une durée initiale de 3 ans.

\section{Déclaration de liens d'intérêts}

$\mathrm{BB}$ a reçu des honoraires de Abbvie, Novartis

$\mathrm{KB}$ a reçu des honoraires de Amgen, Lilly, UCB

VB a reçu des honoraires de Lilly, Amgen, UCB

$\mathrm{RC}$ a reçu des honoraires de Amgen, UCB, Lilly, Arrow ; fonds de recherche Mylan

PG a reçu des honoraires de Alexion, AMGEN, Expanscience, Kyowa Kirin, Lilly, Pfizer

EL a reçu des honoraires en tant qu'expert ou orateur pour les laboratoires Amgen, Abbvie Expanscience, Lilly, MSD, Sublimed et UCB ; soutien financier pour des programmes de recherche des laboratoires Celgene, MSD, UCB

TT a reçu des honoraires en tant qu'expert ou orateur de Amgen, Arrow, Biogen, BMS, Chugai, Expanscience, Gilead, Grunenthal, LCA, Lilly, Medac, MSD, Nordic, Novartis, Pfizer, Sandoz, Sanofi, Theramex, Thuasne, TEVA et UCB et a reçu un soutien financier pour des programmes de recherche de Amgen, Bone Therapeutics, Chugai, MSD, Novartis, Pfizer et UCB, tous non liés à ce manuscrit

B Cortet a reçu des fonds destinés à des activités de recherche ou a participé à des actions ponctuelles de formation ou d'expertise pour les laboratoires Alexion, Amgen, Expanscience, Ferring, Kyowa Kirin, Lilly, MSD, Mylan, Novartis, Roche diagnostics, Theramex, UCB E Legrand, $\mathrm{HB}$ et MD ne déclarent aucun conflit d'intérêt

\section{Remerciements}

Au groupe de lecture : Martine Cohen-Solal, Cyrille Confavreux, Françoise Debiais, JM Feron, Rose-Marie Javier, Christian Marcelli, Pierre Mongiat-Artus, Philippe Orcel, Julien Paccou, Olivier Saint-Lary, Pawel Szulc 


\section{Références}

[1] van Staa TP, Dennison EM, Leufkens HG, Cooper C. Epidemiology of fractures in England and Wales. Bone 2001;29(6):517-22.

[2] Jalava T, Sarna S, Pylkkanen L, Mawer B, Kanis JA, Selby P, et al. Association between vertebral fracture and increased mortality in osteoporotic patients. J Bone Miner Res 2003;18(7):1254-60.

[3] Hasserius R, Karlsson MK, Nilsson BE, Redlund-Johnell I, Johnell O, European Vertebral Osteoporosis S. Prevalent vertebral deformities predict increased mortality and increased fracture rate in both men and women: a 10-year population-based study of 598 individuals from the Swedish cohort in the European Vertebral Osteoporosis Study. Osteoporos Int 2003;14(1):61-8.

[4] Bliuc D, Nguyen ND, Milch VE, Nguyen TV, Eisman JA, Center JR. Mortality risk associated with low-trauma osteoporotic fracture and subsequent fracture in men and women. JAMA 2009;301(5):513-21.

[5] Cumming RG, Klineberg R, Katelaris A. Cohort study of risk of institutionalisation after hip fracture. Aust N Z J Public Health 1996;20(6):579-82.

[6] Benzinger P, Riem S, Bauer J, Jaensch A, Becker C, Buchele G, et al. Risk of institutionalization following fragility fractures in older people. Osteoporos Int 2019;30(7):1363-70.

[7] Borhan S, Papaioannou A, Gajic-Veljanoski O, Kennedy C, loannidis G, Berger C, et al. Incident Fragility Fractures Have a Long-Term Negative Impact on Health-Related Quality of Life of Older People: The Canadian Multicentre Osteoporosis Study. J Bone Miner Res 2019;34(5):838-48.

[8] Bliuc D, Alarkawi D, Nguyen TV, Eisman JA, Center JR. Risk of subsequent fractures and mortality in elderly women and men with fragility fractures with and without osteoporotic bone density: the Dubbo Osteoporosis Epidemiology Study. J Bone Miner Res 2015;30(4):637-46.

[9] Johansson H, Siggeirsdottir K, Harvey NC, Oden A, Gudnason V, McCloskey E, et al. Imminent risk of fracture after fracture. Osteoporos Int 2017;28(3):775-80.

[10] Compton M, Ben Mortenson W, Sale J, Crossman A, Ashe MC. Men's perceptions of living with osteoporosis: a systematic review of qualitative studies. Int J Orthop Trauma Nurs 2019;33:11-7.

[11] Drake MT, Murad MH, Mauck KF, Lane MA, Undavalli C, Elraiyah T, et al. Clinical review. Risk factors for low bone mass-related fractures in men: a systematic review and metaanalysis. J Clin Endocrinol Metab 2012;97(6):1861-70.

[12] Cosman F, de Beur SJ, LeBoff MS, Lewiecki EM, Tanner B, Randall S, et al. Clinician's Guide to Prevention and Treatment of Osteoporosis. Osteoporos Int 2014;25(10):2359-81.

[13] Cauley JA, Cawthon PM, Peters KE, Cummings SR, Ensrud KE, Bauer DC, et al. Risk Factors for Hip Fracture in Older Men: The Osteoporotic Fractures in Men Study (MrOS). J Bone Miner Res 2016;31(10):1810-9.

[14] Briot K, Cortet B, Roux C, Fardet L, Abitbol V, Bacchetta J, et al. 2014 update of recommendations on the prevention and treatment of glucocorticoid-induced osteoporosis. Joint Bone Spine 2014;81(6):493-501.

[15] Briot K, Paccou J, Beuzeboc P, Bonneterre J, Bouvard B, Confavreux CB, et al. French recommendations for osteoporosis prevention and treatment in patients with prostate cancer treated by androgen deprivation. Joint Bone Spine 2019;86(1):21-8. 
[16] Bliuc D, Nguyen TV, Eisman JA, Center JR. The impact of nonhip nonvertebral fractures in elderly women and men. J Clin Endocrinol Metab 2014;99(2):415-23.

[17] Nguyen ND, Pongchaiyakul C, Center JR, Eisman JA, Nguyen TV. Identification of highrisk individuals for hip fracture: a 14-year prospective study. J Bone Miner Res 2005;20(11):1921-8.

[18] Manthripragada AD, O'Malley CD, Gruntmanis U, Hall JW, Wagman RB, Miller PD. Fracture incidence in a large cohort of men age 30 years and older with osteoporosis. Osteoporos Int 2015;26(5):1619-27.

[19] Yang S, Leslie WD, Walld R, Roos LL, Morin SN, Majumdar SR, et al. Objectively-Verified Parental Non-Hip Major Osteoporotic Fractures and Offspring Osteoporotic Fracture Risk: A Population-Based Familial Linkage Study. J Bone Miner Res 2017;32(4):716-21.

[20] Yang S, Leslie WD, Yan L, Walld R, Roos LL, Morin SN, et al. Objectively Verified Parental Hip Fracture Is an Independent Risk Factor for Fracture: a Linkage Analysis of 478,792 Parents and 261,705 Offspring. J Bone Miner Res 2016;31(9):1753-9.

[21] Szulc P, Boutroy S, Vilayphiou N, Chaitou A, Delmas PD, Chapurlat R. Cross-sectional analysis of the association between fragility fractures and bone microarchitecture in older men: the STRAMBO study. J Bone Miner Res 2011;26(6):1358-67.

[22] Genant HK, Wu CY, van Kuijk C, Nevitt MC. Vertebral fracture assessment using a semiquantitative technique. J Bone Miner Res 1993;8(9):1137-48.

[23] Schousboe JT, Lix LM, Morin SN, Derkatch S, Bryanton M, Alhrbi M, et al. Prevalent vertebral fracture on bone density lateral spine (VFA) images in routine clinical practice predict incident fractures. Bone 2019;121:72-9.

[24] Kanis JA, Johnell O, Oden A, Johansson H, McCloskey E. FRAX and the assessment of fracture probability in men and women from the UK. Osteoporos Int 2008;19(4):38597.

[25] Kanis JA, Harvey NC, Cooper C, Johansson H, Oden A, McCloskey EV, et al. A systematic review of intervention thresholds based on FRAX : A report prepared for the National Osteoporosis Guideline Group and the International Osteoporosis Foundation. Arch Osteoporos 2016;11(1):25.

[26] Binkley N, Adler R, Bilezikian JP. Osteoporosis diagnosis in men: the T-score controversy revisited. Curr Osteoporos Rep 2014;12(4):403-9.

[27] Looker AC, Orwoll ES, Johnston CC, Jr., Lindsay RL, Wahner HW, Dunn WL, et al. Prevalence of low femoral bone density in older U.S. adults from NHANES III. J Bone Miner Res 1997;12(11):1761-8.

[28] Cummings SR, Cawthon PM, Ensrud KE, Cauley JA, Fink HA, Orwoll ES, et al. BMD and risk of hip and nonvertebral fractures in older men: a prospective study and comparison with older women. J Bone Miner Res 2006;21(10):1550-6.

[29] Schuit SC, van der Klift M, Weel AE, de Laet CE, Burger H, Seeman E, et al. Fracture incidence and association with bone mineral density in elderly men and women: the Rotterdam Study. Bone 2004;34(1):195-202.

[30] Schousboe JT, Vo TN, Langsetmo L, Taylor BC, Kats AM, Schwartz AV, et al. Predictors of change of trabecular bone score (TBS) in older men: results from the Osteoporotic Fractures in Men (MrOS) Study. Osteoporos Int 2018;29(1):49-59.

[31] Holloway KL, Mohebbi M, Betson AG, Hans D, Hyde NK, Brennan-Olsen SL, et al. Prediction of major osteoporotic and hip fractures in Australian men using FRAX scores adjusted with trabecular bone score. Osteoporos Int 2018;29(1):101-8. 
[32] Orwoll ES, Fino NF, Gill TM, Cauley JA, Strotmeyer ES, Ensrud KE, et al. The Relationships Between Physical Performance, Activity Levels, and Falls in Older Men. J Gerontol A Biol Sci Med Sci 2019;74(9):1475-83.

[33] Szulc P, Montella A, Delmas PD. High bone turnover is associated with accelerated bone loss but not with increased fracture risk in men aged 50 and over: the prospective MINOS study. Ann Rheum Dis 2008;67(9):1249-55.

[34] Bauer DC, Garnero P, Harrison SL, Cauley JA, Eastell R, Ensrud KE, et al. Biochemical markers of bone turnover, hip bone loss, and fracture in older men: the MrOS study. J Bone Miner Res 2009;24(12):2032-8.

[35] Orwoll E, Ettinger M, Weiss S, Miller P, Kendler D, Graham J, et al. Alendronate for the treatment of osteoporosis in men. N Engl J Med 2000;343(9):604-10.

[36] Ringe JD, Dorst A, Faber $\mathrm{H}$, Ibach $\mathrm{K}$. Alendronate treatment of established primary osteoporosis in men: 3-year results of a prospective, comparative, two-arm study. Rheumatol Int 2004;24(2):110-3.

[37] Ringe JD, Faber H, Dorst A. Alendronate treatment of established primary osteoporosis in men: results of a 2-year prospective study. J Clin Endocrinol Metab 2001;86(11):5252-5.

[38] Boonen S, Orwoll ES, Wenderoth D, Stoner KJ, Eusebio R, Delmas PD. Once-weekly risedronate in men with osteoporosis: results of a 2-year, placebo-controlled, doubleblind, multicenter study. J Bone Miner Res 2009;24(4):719-25.

[39] Boonen S, Lorenc RS, Wenderoth D, Stoner KJ, Eusebio R, Orwoll ES. Evidence for safety and efficacy of risedronate in men with osteoporosis over 4 years of treatment: Results from the 2-year, open-label, extension study of a 2-year, randomized, double-blind, placebo-controlled study. Bone 2012;51(3):383-8.

[40] Ringe JD, Faber H, Farahmand P, Dorst A. Efficacy of risedronate in men with primary and secondary osteoporosis: results of a 1-year study. Rheumatol Int 2006;26(5):42731.

[41] Ringe JD, Farahmand P, Faber H, Dorst A. Sustained efficacy of risedronate in men with primary and secondary osteoporosis: results of a 2-year study. Rheumatol Int 2009;29(3):311-5.

[42] Boonen S, Reginster JY, Kaufman JM, Lippuner K, Zanchetta J, Langdahl B, et al. Fracture risk and zoledronic acid therapy in men with osteoporosis. N Engl J Med 2012;367(18):1714-23.

[43] Boonen S, Orwoll E, Magaziner J, Colon-Emeric CS, Adachi JD, Bucci-Rechtweg C, et al. Once-yearly zoledronic acid in older men compared with women with recent hip fracture. J Am Geriatr Soc 2011;59(11):2084-90.

[44] Orwoll E, Teglbjaerg CS, Langdahl BL, Chapurlat R, Czerwinski E, Kendler DL, et al. A randomized, placebo-controlled study of the effects of denosumab for the treatment of men with low bone mineral density. J Clin Endocrinol Metab 2012;97(9):3161-9.

[45] Langdahl BL, Teglbjaerg CS, Ho PR, Chapurlat R, Czerwinski E, Kendler DL, et al. A 24month study evaluating the efficacy and safety of denosumab for the treatment of men with low bone mineral density: results from the ADAMO trial. J Clin Endocrinol Metab 2015;100(4):1335-42.

[46] Orwoll ES, Scheele WH, Paul S, Adami S, Syversen U, Diez-Perez A, et al. The effect of teriparatide [human parathyroid hormone (1-34)] therapy on bone density in men with osteoporosis. J Bone Miner Res 2003;18(1):9-17. 
[47] Lyles KW, Colon-Emeric CS, Magaziner JS, Adachi JD, Pieper CF, Mautalen C, et al. Zoledronic acid and clinical fractures and mortality after hip fracture. $\mathrm{N}$ Engl J Med 2007;357(18):1799-809.

[48] Greenspan SL, Oppenheim DS, Klibanski A. Importance of gonadal steroids to bone mass in men with hyperprolactinemic hypogonadism. Ann Intern Med 1989;110(7):526-31.

[49] Finkelstein JS, Klibanski A, Neer RM, Doppelt SH, Rosenthal DI, Segre GV, et al. Increases in bone density during treatment of men with idiopathic hypogonadotropic hypogonadism. J Clin Endocrinol Metab 1989;69(4):776-83.

[50] Amory JK, Watts NB, Easley KA, Sutton PR, Anawalt BD, Matsumoto AM, et al. Exogenous testosterone or testosterone with finasteride increases bone mineral density in older men with low serum testosterone. J Clin Endocrinol Metab 2004;89(2):503-10.

[51] Basurto L, Zarate A, Gomez R, Vargas C, Saucedo R, Galvan R. Effect of testosterone therapy on lumbar spine and hip mineral density in elderly men. Aging Male 2008;11(3):140-5.

[52] Bhasin S, Brito JP, Cunningham GR, Hayes FJ, Hodis HN, Matsumoto AM, et al. Testosterone Therapy in Men With Hypogonadism: An Endocrine Society Clinical Practice Guideline. J Clin Endocrinol Metab 2018;103(5):1715-44.

[53] Rochira V, Antonio L, Vanderschueren D. EAA clinical guideline on management of bone health in the andrological outpatient clinic. Andrology 2018;6(2):272-85.

[54] Feng YR, Meuleners LB, Fraser ML, Brameld KJ, Agramunt S. The impact of first and second eye cataract surgeries on falls: a prospective cohort study. Clin Interv Aging 2018;13:1457-64.

[55] Bischoff-Ferrari HA, Dawson-Hughes B, Staehelin HB, Orav JE, Stuck AE, Theiler R, et al. Fall prevention with supplemental and active forms of vitamin $D$ : a meta-analysis of randomised controlled trials. BMJ 2009;339:b3692.

[56] Berry SD, Placide SG, Mostofsky E, Zhang Y, Lipsitz LA, Mittleman MA, et al. Antipsychotic and Benzodiazepine Drug Changes Affect Acute Falls Risk Differently in the Nursing Home. J Gerontol A Biol Sci Med Sci 2016;71(2):273-8.

[57] Giangregorio LM, McGill S, Wark JD, Laprade J, Heinonen A, Ashe MC, et al. Too Fit To Fracture: outcomes of a Delphi consensus process on physical activity and exercise recommendations for adults with osteoporosis with or without vertebral fractures. Osteoporos Int 2015;26(3):891-910.

[58] Gillespie LD, Robertson MC, Gillespie WJ, Sherrington C, Gates S, Clemson LM, et al. Interventions for preventing falls in older people living in the community. Cochrane Database Syst Rev 2012(9):CD007146.

[59] Blain H, Masud T, Dargent-Molina P, Martin FC, Rosendahl E, van der Velde N, et al. A Comprehensive Fracture Prevention Strategy in Older Adults: The European Union Geriatric Medicine Society (EUGMS) Statement. J Nutr Health Aging 2016;20(6):64752.

[60] Chastin SF, Mandrichenko O, Helbostadt JL, Skelton DA. Associations between objectively-measured sedentary behaviour and physical activity with bone mineral density in adults and older adults, the NHANES study. Bone 2014;64:254-62.

[61] Langsetmo L, Hitchcock CL, Kingwell EJ, Davison KS, Berger C, Forsmo S, et al. Physical activity, body mass index and bone mineral density-associations in a prospective 
population-based cohort of women and men: the Canadian Multicentre Osteoporosis Study (CaMos). Bone 2012;50(1):401-8.

[62] Holloway-Kew KL, Moloney DJ, Bucki-Smith G, Hyde NK, Brennan-Olsen SL, Timney EN, et al. Sports participation and fracture in older Australian men. Arch Osteoporos 2018;13(1):43.

[63] Michaelsson K, Olofsson H, Jensevik K, Larsson S, Mallmin H, Berglund L, et al. Leisure physical activity and the risk of fracture in men. PLoS Med 2007;4(6):e199.

[64] Menant JC, Close JC, Delbaere K, Sturnieks DL, Trollor J, Sachdev PS, et al. Relationships between serum vitamin $D$ levels, neuromuscular and neuropsychological function and falls in older men and women. Osteoporos Int 2012;23(3):981-9.

[65] Snijder MB, van Schoor NM, Pluijm SM, van Dam RM, Visser M, Lips P. Vitamin D status in relation to one-year risk of recurrent falling in older men and women. J Clin Endocrinol Metab 2006;91(8):2980-5.

[66] Souberbielle JC, Cormier C, Cavalier E, Breuil V, Debiais F, Fardellone P, et al. Vitamin D Supplementation in France in patients with or at risk for osteoporosis: Recent data and new practices. Joint Bone Spine 2020;87(1):25-9.

[67] Bischoff-Ferrari HA, Dawson-Hughes B, Orav EJ, Staehelin HB, Meyer OW, Theiler R, et al. Monthly High-Dose Vitamin D Treatment for the Prevention of Functional Decline: A Randomized Clinical Trial. JAMA Intern Med 2016;176(2):175-83.

[68] Langsetmo L, Barr SI, Berger C, Kreiger N, Rahme E, Adachi JD, et al. Associations of Protein Intake and Protein Source with Bone Mineral Density and Fracture Risk: A Population-Based Cohort Study. J Nutr Health Aging 2015;19(8):861-8.

[69] Langsetmo L, Shikany JM, Cawthon PM, Cauley JA, Taylor BC, Vo TN, et al. The Association Between Protein Intake by Source and Osteoporotic Fracture in Older Men: A Prospective Cohort Study. J Bone Miner Res 2017;32(3):592-600.

[70] Iwamoto J, Uzawa M. Experience with alendronate treatment for 7 years among Japanese men with osteoporosis or osteopenia and clinical risk factors for fractures. Clin Rheumatol 2016;35(1):205-12.

[71] Sugimoto T, Matsumoto T, Hosoi T, Miki T, Gorai I, Yoshikawa H, et al. Three-year denosumab treatment in postmenopausal Japanese women and men with osteoporosis: results from a 1-year open-label extension of the Denosumab Fracture Intervention Randomized Placebo Controlled Trial (DIRECT). Osteoporos Int 2015;26(2):765-74.

[72] Khan AA, Morrison A, Hanley DA, Felsenberg D, McCauley LK, O'Ryan F, et al. Diagnosis and management of osteonecrosis of the jaw: a systematic review and international consensus. J Bone Miner Res 2015;30(1):3-23.

[73] Shane E, Burr D, Abrahamsen B, Adler RA, Brown TD, Cheung AM, et al. Atypical subtrochanteric and diaphyseal femoral fractures: second report of a task force of the American Society for Bone and Mineral Research. J Bone Miner Res 2014;29(1):1-23.

[74] Vasikaran S, Eastell R, Bruyere O, Foldes AJ, Garnero P, Griesmacher A, et al. Markers of bone turnover for the prediction of fracture risk and monitoring of osteoporosis treatment: a need for international reference standards. Osteoporos Int 2011;22(2):391-420.

[75] Moayyeri A, Luben RN, Bingham SA, Welch AA, Wareham NJ, Khaw KT. Measured height loss predicts fractures in middle-aged and older men and women: the EPICNorfolk prospective population study. J Bone Miner Res 2008;23(3):425-32. 
[76] Mikula AL, Hetzel SJ, Binkley N, Anderson PA. Validity of height loss as a predictor for prevalent vertebral fractures, low bone mineral density, and vitamin $D$ deficiency. Osteoporos Int 2017;28(5):1659-65.

[77] Ravaud P, Reny JL, Giraudeau B, Porcher R, Dougados M, Roux C. Individual smallest detectable difference in bone mineral density measurements. J Bone Miner Res 1999;14(8):1449-56. 
Encadré 1 - Contextes étiologiques associés à la survenue d'une perte osseuse et à une augmentation du risque de fracture

- $\quad$ Age $>70$ ans

- $\quad$ IMC $<19 \mathrm{~kg} / \mathrm{m}^{2}$

- Endocrinopathies : hypogonadisme, hypercorticisme, hyperparathyroïdie, hyperthyroïdie, diabète (type 1 et 2), anorexie mentale

- Iatrogènes : corticothérapie, agoniste LH-RH, anti-rétroviraux (ténofovir, inhibiteurs de protéase), chimiothérapie prolongée

- Toxiques : tabagisme actif, maladie alcoolique

- Maladies inflammatoires chroniques : polyarthrite rhumatoïde, spondyloarthrite, maladie de Crohn, rectocolite, infection au VIH

- Hémopathies : mastocytose, gammapathie monoclonale, thalassémie

- $\quad$ BPCO stade $>1$, mucoviscidose

- Insuffisance rénale chronique

- Malabsorption digestive (chirurgie bariatrique, maladie coliaque), hépatopathie chronique

- Hémochromatose

- Maladies associées à une immobilisation prolongée

- Maladies neurologiques avec handicap neuro-sensoriel (Parkinson, séquelles d'hémiplégie...)

- Maladie osseuse constitutionnelle (ostéogénèse imparfaite)

- Hypercalciurie idiopathique (> $300 \mathrm{mg} / 24 \mathrm{~h}$ ) 
Encadré 2 - Circonstances cliniques au cours desquelles il est recommandé de réaliser une densitométrie osseuse (* répondent aux conditions de remboursement)

- Découverte ou confirmation radiologique d'une fracture vertébrale (déformation du corps vertébral) sans contexte traumatique ni tumoral évident*

- Antécédent personnel de fracture périphérique survenue sans traumatisme majeur (exclues fracture du crâne, des orteils, des doigts, du rachis cervical) *

- Corticothérapie systémique (de préférence au début) prescrite pour une durée d'au moins 3 mois consécutifs, à une dose $>7,5 \mathrm{mg} / \mathrm{j}$ d'équivalent prednisone*

- Hypogonadisme prolongé (incluant la castration chirurgicale ou médicamenteuse par traitement prolongé par analogue de la $\mathrm{Gn}-\mathrm{RH})$ *

- Hyperthyroïdie évolutive non traitée*

- Hypercorticisme endogène*

- Hyperparathyroïdie primitive*

- Ostéogénèse imparfaite*

- Chutes répétées

- Infection par le VIH

- Maladies pulmonaires chroniques, BPCO stade > 1

- Rhumatismes inflammatoires chroniques 


\section{Encadré 3 - Définitions}

\section{* Ostéoporose}

Il n'existe pas de définition consensuelle de l'ostéoporose masculine mais il est admis d'utiliser la définition féminine. Ainsi, l'ostéoporose se définit par

- la présence d'une ou plusieurs fracture(s) par fragilité osseuse

- et/ou la découverte d'une DMO basse (définie par un T-score $\leq-2,5$ ) dans un contexte clinique et/ou biologique associé à une fragilité osseuse (exemples : corticothérapie prolongée, maladie alcoolique, hypogonadisme, BPCO)

* Fracture sévère

Les fractures dites sévères sont associées à un excès de mortalité ; ce sont les fractures de l'extrémité supérieure du fémur, de l'extrémité supérieure de l'humérus, des vertèbres, du bassin, de la diaphyse fémorale et du fémur distal, des trois côtes simultanées et du tibia proximal

* Fracture majeure

Terme utilisé dans le calcul du score $\mathrm{FRAX}^{\circledR}$, qui regroupe les fractures de l'extrémité supérieure du fémur, de humérus, du poignet et les fractures vertébrales cliniques 


\section{Encadré 4 - Dans quelles circonstances référer un patient à un spécialiste de la pathologie} osseuse ?

- La survenue d'une fracture sévère chez un homme avec des T-scores tous > -1

- En présence d'une contre-indication au traitement (ex : insuffisance rénale)

- Si le patient ne remplit pas les conditions d'arrêt de traitement après une séquence thérapeutique

- La découverte d'une ostéoporose ou d'une fracture par fragilité osseuse chez un homme jeune dans une situation clinique inhabituelle 
Tableau 1 - Effet des traitements sur le risque de fracture et la DMO

\begin{tabular}{|c|c|c|c|c|c|c|}
\hline Traitement & Etude & Durée & Objectifs & DMO & Fractures & Commentaires \\
\hline Alendronate & $\begin{array}{l}\text { Orwoll [35] } \\
\text { Ringe }[36,37]\end{array}$ & 2 ans & $\begin{array}{l}1^{\text {aire }}: \text { DMO RL et CF } \\
2^{\text {aire }}: \text { FV Morpho et } \\
\text { FNV }\end{array}$ & $\begin{array}{l}\mathrm{RL}+7,1 \% \text { vs } 1,8 \% \\
\mathrm{CF}+2,5 \% \text { v } 0 \\
\mathrm{RL}+11,5 \% \text { vs } 3,5 \%(<0,01) \\
\mathrm{CF}+5,8 \% \text { vs } 2,3 \%(<0,01)\end{array}$ & $\begin{array}{l}\text { FV : } 0,8 \% \text { alendronate vs } 7,1 \% \text { placebo } \\
\text { OR } 0,35(0,10-1,24) \\
\text { FNV : } 4,1 \% \text { vs } 5,3 \% \\
\text { OR } 0,77(0,23-2,60) \\
\text { FV : } 10,3 \% \text { vs } 24,2 \%(0,04) \\
\text { OR } 0,36(0,14-0,94) \\
\text { FNV : } 8,8 \% \text { vs } 12,1 \% \\
\text { OR } 0,70(0,23-2,14)\end{array}$ & $\begin{array}{l}\mathrm{ERC}, 241 \text { hommes } \\
\text { Alendronate } 10 \mathrm{mg} / \text { jour } \mathrm{n}=146 \text { vs placebo } \mathrm{n}=95+500 \mathrm{mg} \text { calcium }+400-450 \mathrm{UI} \text { vitamine } \mathrm{D} \\
\text { Résultats identiques quel que soit le taux de testostérone initial } \\
\text { Randomisée, contrôlée, ouverte, } 134 \text { hommes } \\
\text { (Alendronate } 10 \mathrm{mg} / \text { jour } \mathrm{n}=68 \text { vs } 1 \text {-alfacalcidol } 1 \mu \mathrm{g} / \text { jour } \mathrm{n}=66)+500 \mathrm{mg} \text { calcium }\end{array}$ \\
\hline Risédronate & Boonen $[38,39]$ & 2 ans & $\begin{array}{l}1^{\text {aire }}: \text { Nouvelles FV } \\
\text { DMO RL, CF, HT }\end{array}$ & $\begin{array}{l}\mathrm{RL}+6 \% \text { vs } 1,4 \%(<0,01) \\
\mathrm{RL}+6,5 \% \text { vs } 2,2 \%(<0,01) \\
\mathrm{CF}+3,2 \% \text { vs } 0,6 \%(<0,01) \\
\mathrm{HT}+4,4 \% \text { vs }+0,4 \%(<0,01)\end{array}$ & $\begin{array}{l}\text { FV : } 9,2 \% \text { vs } 23,6 \%(0,002) \\
\text { OR } 0,40(0,10-0,88) \\
\text { FNV } \\
\text { OR } 0,59(0,28-1,24)\end{array}$ & $\begin{array}{l}\text { ERC, } 284 \text { hommes } \\
\text { (Risédronate } 35 \mathrm{mg} \mathrm{n}=191 \text { vs placebo } \mathrm{n}=93)+1000 \mathrm{mg} \text { calcium }+400-500 \mathrm{UI} \text { vitamine } \mathrm{D} \\
\text { Essai ouvert, } 316 \text { hommes } \\
\text { Risédronate } 5 \mathrm{mg} / \mathrm{jour}+1000 \mathrm{mg} \text { calcium }+800 \mathrm{UI} \text { vitamine } \mathrm{D}(\mathrm{n}=158) \text { vs } 500 \mathrm{mg} \text { calcium }+ \\
1 \mu \mathrm{g} \text { alfacalcidol }: \mathrm{si} \text { FV à l'inclusion ou } 800 \mathrm{mg} \text { calcium }+1000 \mathrm{UI} \text { vitamine } \mathrm{D}: \text { si pas de FV } \\
\text { à l'inclusion }(\mathrm{n}=158)\end{array}$ \\
\hline $\begin{array}{l}\text { Acide } \\
\text { zolédronique }\end{array}$ & $\begin{array}{l}\text { Boonen [42] } \\
\text { Boonen [43] }\end{array}$ & 3 ans & 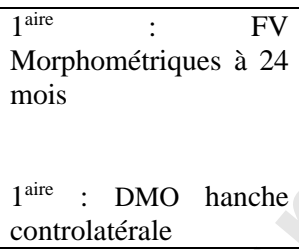 & $\begin{array}{l}\text { RL }+7,7 \% \text { vs } 1,6 \%(<0,01) \\
\text { HT M12: }+2 \% \\
\text { HT M24 : }+3,8 \%\end{array}$ & $\begin{array}{l}\text { FV : } 1,6 \% \text { vs } 4,9 \%(0,04) \\
\text { RR } 0,33(0,16-0,70) \\
1^{\text {ere }} \text { fracture clinique à M24 : } \\
7,5 \% \text { vs } 8,7 \% \mathrm{HR}=0,85, \mathrm{p}=0,64\end{array}$ & $\begin{array}{l}\text { ERC, } 1199 \text { hommes } \\
(\text { Ac zol vs placebo) }+1000-1500 \mathrm{mg} \text { calcium }+800-1200 \mathrm{IU} \text { vitamine } \mathrm{D} \\
\text { Perf ac zol à } 0 \text { et } 1 \text { an } \\
\text { ERC, } 508 \text { hommes après fracture de hanche } \\
(\text { ac zol } \mathrm{n}=248 \text { vs pbo } \mathrm{n}=260)\end{array}$ \\
\hline Dénosumab & $\begin{array}{l}\text { Orwoll [44] } \\
\text { Langdahl [45] }\end{array}$ & 2 ans & $1^{\text {aire }}:$ DMO RL & $\begin{array}{l}\text { RL }+5,7 \% \text { vs } 0,9 \% \\
\mathrm{CF}+2,1 \% \text { vs } 0 \% \\
\mathrm{HT}+2,4 \% \text { vs } 0,3 \% \\
\mathrm{RL}+8 \% \\
\mathrm{CF}+3,4 \% \\
\mathrm{HT}+3,4 \%\end{array}$ & Très peu d'évènements (non significatif) & $\begin{array}{l}\text { (Denosumab } 60 \mathrm{mg} / 6 \mathrm{mois} \text { vs placebo })+1000 \mathrm{mg} \text { calcium }+800 \mathrm{UI} \text { vitamine } \mathrm{D} \\
2^{\mathrm{e}} \text { année en ouvert }\end{array}$ \\
\hline Tériparatide & Orwoll [46] & 2 ans & $1^{\text {aire }}:$ DMO RL & $\begin{array}{l}\text { RL: } \\
+5,9 \%(20 \mu \mathrm{g}) \\
+9,0 \%(40 \mu \mathrm{g})(\mathrm{p}<0.001 v \mathrm{p} \mathrm{pbo}) \\
\mathrm{CF} \\
+1,5 \%(20 \mu \mathrm{g}) \\
+2,9 \%(40 \mu \mathrm{g} ; \mathrm{p}<0.001)\end{array}$ & Pas de données & $\begin{array}{l}\text { ERC, } 437 \text { hommes } \\
\text { Tériparatide } 20 \mu \mathrm{g} / \mathrm{jour}(\mathrm{n}=151) \text { ou } 40 \mu \mathrm{g} / \mathrm{jour}(\mathrm{n}=139) \text { vs placebo }+1000 \mathrm{mg} \text { calcium }+400 \text { - } \\
1200 \text { UI vitamine } \mathrm{D}(\mathrm{n}=147) \\
\text { Etude arrêtée précocement (médiane } 11 \text { mois) }\end{array}$ \\
\hline
\end{tabular}

Abréviations : DMO = densité minérale osseuse, $\mathrm{FV}=$ fracture vertébrale, $\mathrm{FNV}=$ fracture non vertébrale, $\mathrm{RL}=$ rachis lombaire, $\mathrm{CF}=\mathrm{col}$ fémoral, $\mathrm{HT}=$ hanche totale, $\mathrm{ERC}$ :

étude randomisée contrôlée 
Tableau 2 - Indications thérapeutiques dans l'ostéoporose masculine

\begin{tabular}{|l|l|l|l|}
\hline & $\begin{array}{l}\text { Fracture sévère } \\
\text { (Vertèbre, bassin, } \\
\text { extrémité supérieure du } \\
\text { fémur, fémur distal, } \\
\text { humérus proximal) }\end{array}$ & $\begin{array}{l}\text { Fracture non sévère } \\
\text { (Poignet, cheville) }\end{array}$ & $\begin{array}{l}\text { Pas de fracture mais facteurs de } \\
\text { risque de fracture par fragilité } \\
\text { osseuse* }\end{array}$ \\
\hline T-score $>\mathbf{- 1}$ & Avis spécialisé & Pas de traitement & Pas de traitement \\
\hline $\mathbf{T} \leq-\mathbf{1}$ et $>\mathbf{- 2}$ & Traitement & Avis spécialisé & Pas de traitement \\
\hline T-score $\leq-\mathbf{2}$ & Traitement & Traitement & Avis spécialisé \\
\hline T-score $\leq-\mathbf{3}$ & Traitement & Traitement & Traitement \\
\hline
\end{tabular}

* La prise en charge de l'ostéoporose dans les contextes bien spécifiques de la corticothérapie prolongée et du traitement du cancer de la prostate par hormonothérapie anti-androgénique fait l'objet de recommandations spécifiques publiées par ailleurs [14, 15] 